\title{
Evaluation of the Thermal Interface Crack Problem in Packaging Structure
}

\author{
Fengnan Guo ${ }^{1, a}$, Jianwei Cui ${ }^{1}$, Yuanhe Wang ${ }^{1}$, Yu Liu ${ }^{2}$, Licheng Guo ${ }^{3}$ \\ ${ }^{1}$ The $41^{\text {st }}$ Institute of No.6 Academy China Aerospace Science \& Industry Corp. \\ Hohhot, 010010, China \\ ${ }^{2}$ Military Agent's Room, Baotou, 014000, China \\ ${ }^{3}$ The Department of Astronautic Science and Mechanics, Harbin Institute of Technology, Harbin, \\ 150001, China \\ agf-n@163.com
}

Keywords: Packaging structure, interaction energy integral, thermal stress intensity factors, interface crack.

Abstract. In this paper, the interaction integral method is developed to study the thermal fracture mechanics of packaging structures. The present interaction integral can be proved to be domain-independent for the packaging structures with complex interfaces. The mixed-mode thermal stress intensity factors (TSIFs) of the packaging structure can be obtained using the present method. The domain-independence of the present method is verified. The thermal fracture problems of flip-chip packaging with complex interfaces are investigated.

\section{Introduction}

Packaging structure plays an important role in the current development of electronic components. Thermal fracture is the major failure model of packaging materials. It absorbed great attention from the international research community. The study of the thermal fracture problems of package structures is important for the designment, optimization and reliable application of package structures.

The mechanism of the interfacial fracture problems in packaging structure under thermal loading is studied by many researchers. Desai and Whitenack [1] reviewed some models and for thermomechanical stress analysis in electronic packaging structure and identified the capabilities and limitations of them. Nied [2] investigated the interface fracture problems of electronic packaging. Wang et al. [3] studied the interfacial fracture toughness in a flip-chip package subjected to a constant concentrated line load and a bimaterial system under thermal loading condition.

In this paper, the thermal interface crack problem of a flip-chip packaging is investigated and the thermal stress intensity factors (TSIFs) are evaluated by means of an interaction integral.

\section{Simplified model of flip-chip packaging}

In this paper, a simplified model of a flip-chip packaging structure is adopted for evaluating the thermal interface crack problem. As shown in Fig. 1, there is a simplified model which ignoring some tiny structures such as lead. Due to the symmetry, we take the left half of this model to analyze. The model size can be seen in Fig. 2 and the material properties are shown in Table 1 [4].

Table 1 TSIFs for different integral domains for a nonhomogeneous material plate

\begin{tabular}{cccc}
\hline Materials & Elastic Modulus(Mpa) & CTE $\left(/^{\circ} \mathrm{C}\right)$ & \multicolumn{2}{c}{ Poisson's Ratio } \\
\hline Silicon Die & 13100 & 2.8 & 0.3 \\
\hline Solder & 30674 & 21 & 0.35 \\
\hline Encapsulant & 6900 & 29 & 0.3 \\
\hline FR-4(Substrate) & 22000 & 18 & 0.28 \\
\hline
\end{tabular}


There is an interface crack located at the left side of the mode. If the crack tip is near the interface of the Solder and Encapsulant, the integral domain will contain three complex interfaces. It is difficult to obtain the crack tip TSIFs using J-integral because the J-integral lost the path-independent in this condition. So we use the interaction integral to deal with this complex situation.

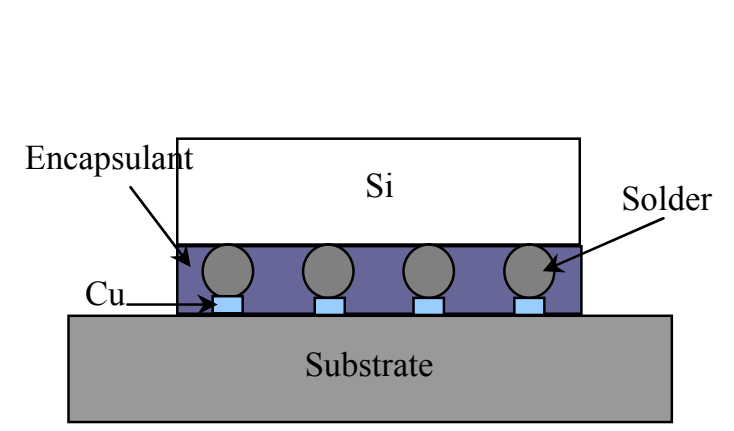

Figure 1. Simplified model of a flip-chip packaging.

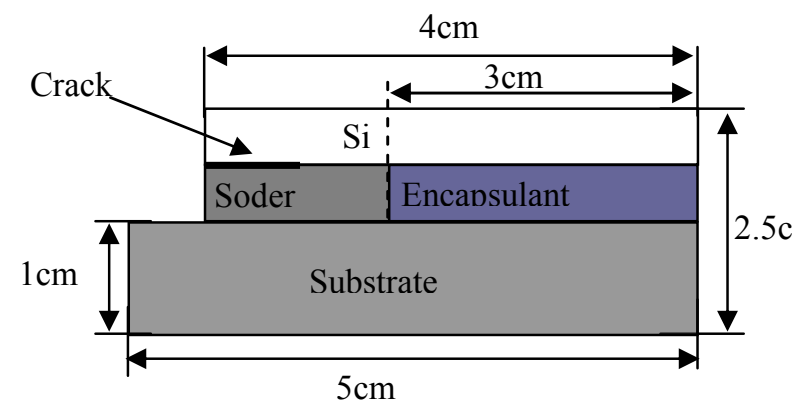

Figure 2. Size of the simplified model with a crack

The interaction integral [5] can be expressed as

$$
I=\lim _{\Gamma_{0} \rightarrow 0} \oint_{\Gamma_{2}}\left(\sigma_{i j}^{a u x} u_{i, 1}+\sigma_{i j} u_{i, 1}^{a u x}-\sigma_{i k}^{a u x} \varepsilon_{i k}^{m} \delta_{1 j}\right) m_{j} q d \Gamma
$$

where $\varepsilon_{i k}^{m}$ is the mechanical strain and it can be expressed as $\varepsilon_{i k}^{m}=\varepsilon_{i k}^{\text {total }}+\varepsilon_{i k}^{\text {thermal }}$ with $\varepsilon_{i k}^{\text {total }}$ as total strain and $\varepsilon_{i k}^{\text {thermal }}$ as the thermal strain. The integral counter $\Gamma_{2}=\Gamma_{1}+\Gamma_{c}^{+}-\Gamma_{0}+\Gamma_{c}^{-}$as shown in Fig.3. And $m_{j}$ is the outward normal vector to the contour $\Gamma_{2}$.

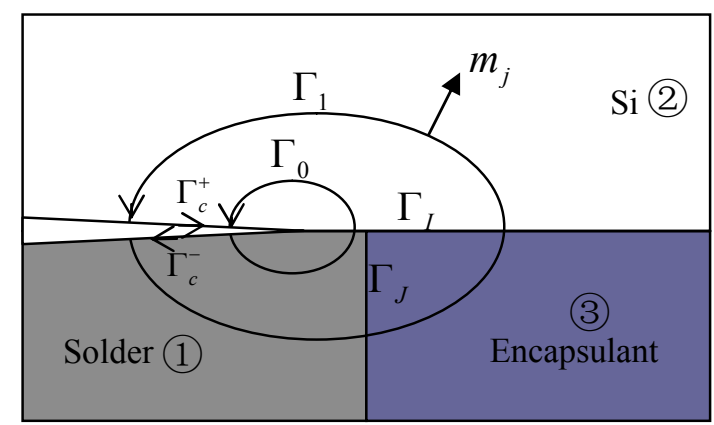

Figure 3. Integral domain and the contour integral for interface crack

For interface crack problem, the interaction integral can be derived as the following form using the divergence theorem from.

$$
\begin{aligned}
I= & \int_{A}\left(\sigma_{i j}^{a u x} u_{i, 1}+\sigma_{i j} u_{i, 1}^{a u x}-\sigma_{i k}^{a u x} \varepsilon_{i k}^{m} \delta_{1 j}\right) q_{, j} d A \\
& +\int_{A}\left(\sigma_{i j}^{a u x} u_{i, j 1}+\sigma_{i j} u_{i, j 1}^{a u x}-\sigma_{i j, 1}^{a u x} \varepsilon_{i j}^{m}-\sigma_{i j}^{a u x} \varepsilon_{i j, 1}^{m}\right) q d A+I_{\Gamma_{I}}
\end{aligned}
$$

where the interface integral can be written as

$$
I_{\Gamma_{I}}=\int_{\Gamma_{I}}\left\{\left(\sigma_{i j}^{a u x} u_{i, 1}+\sigma_{i j} u_{i, 1}^{a u x}-\sigma_{i k}^{a u x} \varepsilon_{i k}^{m} \delta_{1 j}\right)^{2}-\left(\sigma_{i j}^{a u x} u_{i, 1}+\sigma_{i j} u_{i, 1}^{a u x}-\sigma_{i k}^{a u x} \varepsilon_{i k}^{m} \delta_{1 j}\right)^{(1)}\right\} m_{j} q d \Gamma
$$

According to the definitions of the auxiliary fields [6], the interface integral can be obtained as

$$
I_{\Gamma_{I}}=0
$$

So, the interaction energy integral for the thermal interface problems can be written as

$$
\begin{aligned}
I= & \int_{A}\left(\sigma_{i j}^{a u x} u_{i, 1}+\sigma_{i j} u_{i, 1}^{a u x}-\sigma_{i k}^{a u x} \varepsilon_{i k}^{m} \delta_{1 j}\right) q_{, j} d A \\
& +\int_{A}\left(\sigma_{i j}\left(S_{i j k l}^{t i p}-S_{i j k l}(x)\right) \sigma_{k l, 1}^{a u x}\right) q d A+\int_{A} \sigma_{i i}^{a u x}\left[\alpha_{, 1}\left(T-T_{0}\right)+\alpha T_{, 1}\right] q d A
\end{aligned}
$$

When the crack intersects another interface $\Gamma_{J}$, a liner integral should be added to the interaction integral express [7], that is 


$$
\begin{aligned}
I= & \int_{A}\left(\sigma_{i j}^{a u x} u_{i, 1}+\sigma_{i j} u_{i, 1}^{a u x}-\sigma_{i k}^{a u x} \varepsilon_{i k}^{m} \delta_{1 j}\right) q_{, j} d A \\
& +\int_{A}\left(\sigma_{i j}\left(S_{i j k l}^{t i p}-S_{i j k l}(x)\right) \sigma_{k l, 1}^{a u x}\right) q d A+\int_{A} \sigma_{i i}^{a u x}\left[\alpha_{, 1}\left(T-T_{0}\right)+\alpha T_{, 1}\right] q d A \\
& +\int_{\Gamma_{J}}\left[\sigma_{i i}^{a u x}\left(\alpha^{(3)}-\alpha^{(1)}\right)\left(T-T_{0}\right) \cos \theta\right] q d \Gamma
\end{aligned}
$$

The relationships between the interaction integral and the TSIFs are

$$
\begin{array}{lll}
K_{I}=E_{\text {tip }}^{\prime} I / 2 & \text { when } & K_{I}^{a u x}=1, K_{I I}^{a u x}=0 \\
K_{I I}=E_{\text {tip }}^{\prime} I / 2 & \text { when } & K_{I}^{a u x}=0, K_{I I}^{a u x}=1
\end{array}
$$

where $E_{t i p}^{\prime}=E_{t i p} /\left(1-v_{t i p}{ }^{2}\right)$ for generalized plane stain and $E_{t i p}^{\prime}=E_{t i p}$ for plane stress. We can obtain the TSIFs easily if the interaction integral $I$ is determined.

\section{Examples and discussions}

As shown in Fig. 2, the model contains a edge interface crack of length $a$. In this example, one-dimensional thermal conduction is assumed (along $x$-direction). The temperatures at the top and the bottom surface are denoted by $T_{1}$ and $T_{2}$, respectively. The temperature boundary conditions are selected as $T_{1}=T_{2}=100^{\circ} \mathrm{C}$, where $T_{0}=0^{\circ} \mathrm{C}$ represents the initial temperature. The displacement boundary conditions are prescribed such that $u_{x}=0$ along the right edge. The normalized factor $K_{0}=E_{0} \alpha_{0}\left(T_{1}-T_{0}\right) \sqrt{\pi a} /(1-v)$; generalized plane strain.

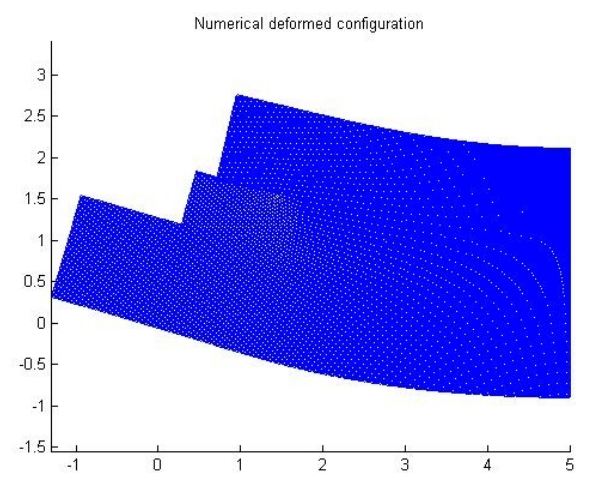

Figure 4. Deformation of the flip-chip packaging under thermal loading.

Firstly, we set the crack length $a$ as $0.75 \mathrm{~cm}$. Fig. 4 shows the deformation of the model (Multiplier 100). It can be seen that the Si layer and the Soder layer is delaminating because of the existence of crack.

In order to verify the domain-independence of the interaction integral, four domains $\left(R_{c} / R_{\text {tip }}=8,16\right.$, $20,24)$ are selected. Where $R_{\text {tip }}$ is the edge length of the elements at the crack tip and $R_{c}$ is the radius of the referenced circular contour $\mathrm{C}_{I}$. Table 2 shows the TSIFs computed for different integral domains. In Table 2, the maximum relative error of the TSIFs computed for different $\mathrm{R}_{\mathrm{c}} / \mathrm{R}_{\text {tip }}$ is less than $0.3 \%$. Thus, the domain-independence of the present method can be demonstrated.

Table 2 TSIFs for different integral domains for a Flip chip packaging

\begin{tabular}{ccc}
\hline Integral domain $\mathrm{R}_{\mathrm{c}} / \mathrm{R}_{\text {tip }}$ & $K_{1}$ & $K_{2}$ \\
\hline 8 & -15.7252 & 20.0034 \\
\hline 16 & -15.6865 & 20.0017 \\
\hline 20 & -15.7069 & 20.0114 \\
\hline 24 & -15.7077 & 20.0118 \\
\hline Relative error & $<0.3 \%$ & $<0.1 \%$ \\
\hline
\end{tabular}


Then we will discuss the influence of the discontinuity material properties on the TSIFs. The temperature boundary conditions are selected as $T_{1}=100^{\circ} \mathrm{C}, T_{2}=10^{\circ} \mathrm{C}$ and $T_{0}=0^{\circ} \mathrm{C}$. Fig. 4 shows the TSIFs varies with the different crack length. It can be observed that when the crack tip across the interface of the Solder and Encapsulant, the TSIFs have a great change. It can be that the mismatch of material properties can affect the TSIFs dramatically.

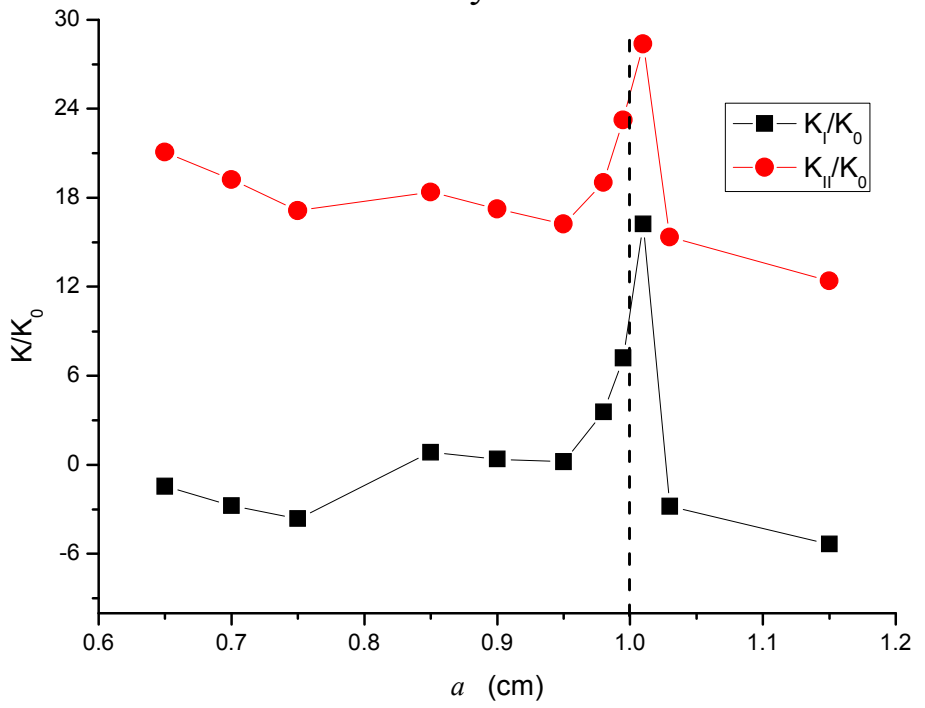

Figure 5. Normalized TSIFs in flip-chip packaging vary with the crack length.

\section{Summary}

The interaction integral method can be used to obtain the TSIFs of the flip-chip packaging under thermal loading. The interaction energy integral can be proved to be domain-independent for the interface crack problems even when the integral domain intersecting another interface with discontinuous thermomechanical properties. Due to this, some thermal fracture problems in electronic packaging with complex structures can be solved effectively with high efficiency. The domain-independence of the interaction energy integral is verified. An example is proposed to study the influence of the discontinuous material properties on the TSIFs. It can be found that the material properties have a great effect on the TSIFs.

\section{References}

[1] C.S. Desai R. Whitenack: J Electron Packaging, Vol. 123 (2011), p.19-33.

[2] H.F. Nied. IEEE T Device Mat Re, Vol. 3 (2003), p.129-143.

[3] J.J. Wang, D.Q. Zou, M.F. Lu, W. Ren and S. Liu: Eng Fract Mech, Vol. 64 (1999), p.781-797.

[4] J.H.L. Pang, D.Y.R. Chong and T.H. Low: IEEE T Device Mat Re, Vol. 24 (2001), p.705-712.

[5] J.H. Kim, A. KC. J. Appl. Mech. Vol. 75 (2008), p. 1-11.

[6] H.J. Yu, L.Z. Wu, L.C. Guo, S.Y. Du, Q.L. He: Int J Solids Struct, Vol. 46 (2009), p. 3710-3724.

[7] L.C. Guo, F.N. Guo, H.J. Yu, L. Zhang: Int J Solids Struct, Vol. 49 (2012), p. 355-365. 